\title{
STRUCTURE THEOREM FOR COMMUTATORS OF OPERATORS
}

\author{
BY ARLEN BROWN AND CARL PEARCY
}

Communicated by P. R. Halmos, July 13, 1964

If $\mathfrak{H}$ is a separable (complex) Hilbert space, and $A$ is a (bounded, linear) operator on $\mathcal{F}$, then $A$ is a commutator if there exist operators $B$ and $C$ on $F$ such that $A=B C-C B$. It was shown by Wintner [8] and also by Wielandt [7] that no nonzero scalar multiple of the identity operator $I$ on $\mathcal{H}$ is a commutator, and this was improved by Halmos [5] who showed that no operator of the form $\lambda I+C$ is a commutator, where $\lambda \neq 0$ and $C$ is a compact operator. The purpose of this note is to announce the following theorem and give some indication of its proof. Details of the results described below will appear elsewhere [2].

Theorem. An operator $A$ on a separable Hilbert space $\mathfrak{H C}$ is a commutator if and only if $A$ is not of the form $\lambda I+C$ where $\lambda \neq 0$ and $C$ is a compact operator.

This theorem furnishes the solution to several problems concerning commutators posed by Halmos in [4] and [5]. In particular it is interesting to note that the identity operator is the limit in the norm of commutators and that there exists a commutator whose spectrum consists of the number 1 alone.

INDICATION OF THE PROOF. We must show that every operator that is not of the form $\lambda I+C$, with $\lambda \neq 0$ and $C$ compact, is a commutator. These operators fall naturally into two classes; viz., the class of compact operators, which was shown to consist entirely of commutators in [1], and the class consisting of all operators that cannot be written in the form $\lambda I+C$ for any scalar $\lambda$ (0 or not) and compact $C$. We denote this latter class by $(F)$, and the first problem is to obtain a more usable characterization of the operators of this class. To this end we define for an arbitrary operator $T$ on $\mathfrak{T}$ the function

$$
\eta_{T}(x)=\|T x-(T x, x) x\|, \quad x \in \mathfrak{F},\|x\|=1,
$$

and denote by $\eta_{T}(\mathscr{T T})$ the supremum over the subspace $\mathscr{M C} \subset \mathcal{F}$ of $\eta_{T}(x)$.

Proposition 1. An operator $T$ is of type $(F)$ if and only if inf $\eta_{T}(\mathfrak{T T})$ $>0$ where the infimum is taken over all cofinite-dimensional subspaces TT of $\mathfrak{H C}$. 
This proposition may then be employed to yield a "standard form" for operators of type $(F)$.

Proposition 2. Every operator of type $(F)$ is similar to an operator of the form

$$
\left(\begin{array}{lll}
A_{11} & A_{12} & 0 \\
A_{21} & A_{22} & I \\
A_{31} & A_{32} & 0
\end{array}\right)
$$

acting in the usual fashion on a Hilbert space $\Re \oplus \Re \oplus \Re$. (The $A_{i j}$ are, of course, operators on $\mathfrak{K}$.)

It is easily seen from this that to complete the proof it suffices to show that every $2 \times 2$ operator matrix of the form

$$
\left(\begin{array}{ll}
A & U \\
B & 0
\end{array}\right)
$$

where $U$ is an isometry with infinite deficiency, is a commutator. This is accomplished by making a fairly intricate sequence of computations involving $2 \times 2$ matrices with operator entries. A central tool used in this argument is the result [6] that every operator with an infinite-dimensional null space is a commutator.

We note in conclusion that the restriction to separable spaces in the statement of the above theorem is for the sake of simplicity only; analogous results hold for an arbitrary infinite-dimensional Hilbert space.

\section{BIBLIOGRAPHY}

1. Arlen Brown, P. R. Halmos and Carl Pearcy, Commutators of operators on Hilbert space, Canad. J. Math. (to appear).

2. Arlen Brown and Carl Pearcy, Structure of commutators of operators (to appear).

3. P. R. Halmos, Commutators of operators, Amer. J. Math. 74 (1952), 237-240.

4. - Commutators of operators. II, Amer. J. Math. 76 (1954), 191-198.

5. — A glimpse into Hilbert space, Lectures in Mathematics, Wiley, New York, 1963.

6. Carl Pearcy, On commutators of operators on Hilbert space, Proc. Amer. Math. Soc. (to appear).

7. H. Wielandt, Über die Unbeschränktheit der Operatoren der Quantenmechanik, Math. Ann. 121 (1949), 21.

8. A. Wintner, The unboundedness of quantum-mechanical matrices, Phys. Rev. 71 (1947), 738-739.

UNIVERSITY OF MichigaN 